\title{
Evaluation of modified MRS media for the selective enumeration of Lactobacillus casei
}

\author{
Ifra Farzand ${ }^{1}$, Sajjad Ur Rahman ${ }^{1 *}$, Sanaullah Sajid ${ }^{1}$ and Sehrish \\ Nayab ${ }^{1}$ \\ 1. Institute of Microbiology, University of Agriculture, Faisalabad-Pakistan \\ *Corresponding author's email: sajjadur@gmail.com \\ Citation \\ Ifra Farzand, Sajjad Ur Rahman, Sanaullah Sajid and Sehrish Nayab. Evaluation of modified MRS media for the \\ selective enumeration of Lactobacillus casei. Pure and Applied Biology. Vol. 10, Issue 1, pp194-198. \\ http://dx.doi.org/10.19045/bspab.2021.100020
}

\begin{tabular}{llll}
\hline \hline Received: 01/06/2020 & Revised: 24/08/2020 & Accepted: 25/08/2020 & Online First: 16/09/2020 \\
\hline \hline
\end{tabular}

\begin{abstract}
Lactobacillus is an extensive group of Gram-positive, catalase negative and non-spore forming organism belongs to Lactobacillaceae family. $L$. case $i$ is an obligate fermentative bacterium with complex nutritional requirements. It has its novel importance as a bio-preservative agent and enhancing food quality. Lactobacillus casei is Generally Recognized as Safe (GRAS). For the isolation and identification, cultural media is one of the most important tools and the methods to distinguish between Lactobacillus casei and Lactobacillus lactis are based upon various biochemical tests. In general, MRS media is used for the isolation of Lactobacillus species but we have developed the modified selective medium that utilizes the calcium gluconate. Modified media involves a $\mathrm{pH}$ indicator, differences in $\mathrm{pH}$ can be observed by means of detectable color change. An intervention study was conducted to confirm the capability of modified MRS medium to detect $L$. casei and $L$. lactis recovered from dairy samples. We were able to enumerate circular medium sized predominant yellowish colored colonies morphologically similar to Lactobacillus casei while the growth of Lactobacillus lactis was inhibited. These colonies were then subjected to PCR to identify L. casei.
\end{abstract}

Keywords: GRAS; L. casei; L. lactis; MRS; PCR

\section{Introduction}

Fermented milk products are made of milk under controlled conditions for producing flavor and acidity at desired level. Organisms used in fermentation mostly belong to Lactic Acid Bacteria (LAB). LAB have specific metabolic, physiological and morphological characteristics. Lactic Acid Producing Bacteria most commonly present in nature as well as necessary organisms in raw milk and fermented yogurt. LAB are Gram positive organisms playing important role in the fermentation of food products. LAB usually are non-sporulating and non-motile organisms which produce lactic acid as an important end product [1]. Production of lactic acid is the most important quality of LAB because they prevent the attachment, proliferation and settlement of harmful bacteria. Many components are compulsory for the growth of LAB like nucleic acid, carbohydrates, peptides, vitamins and amino acids [2]. Among lactic acid forming organisms Lactobacillus are the most dominant organisms consist of various Gram positive, rod shaped, non-pigmented, nonspore forming, microaerophilic to strictly anaerobic organisms [3-5].

Lactobacillus casei (L. casei) is considered as the main habitat of gut microflora and is present in the variety of commercial food products that are fermentative in nature 
[6]. L. casei has shortest growing time among all organisms belongs to Lactobacillus genus [7]. There is no selective media present for the isolation of entire LAB. Isolation media may be altered by change in concentration of reagents which are inhibitory in nature, modifying $\mathrm{pH}$ value, adjustment in temperature and time. MRS medium is well known medium for culturing LAB having $\mathrm{pH} 5.7$ to 6.2 [8].

The objective of this research was to optimize the chemically defined medium and to test the suitability of modified MRS medium for the selective enumeration of $L$. casei. The efficacy of modified MRS medium for the selective enumeration of $L$. casei was assessed using commercial yogurt and fermented milk obtained from local supermarkets.

\section{Materials and Methods}

\section{Isolation of Lactobacillus}

For the isolation of Lactobacillus, commercial yogurt and dairy samples were collected from local market. Samples were serially diluted as reported by Noori and Jafari [9]. Test tubes were labeled with dilution factors as $10^{-1}$ and $10^{-2}$. In each test tube $4.5 \mathrm{ml}$ of normal saline was added. With the help of sterilized pipette, $0.5 \mathrm{ml}$ of sample was inoculated in test tube (labeled with $10^{-1}$ ) and mixed thoroughly. To make dilutions $0.5 \mathrm{ml}$ of sample from $10^{-1}$ test tube was shifted to the next test tube, labeled as $10^{-2}$. Both test tubes were incubated at $37^{\circ} \mathrm{C}$ anaerobically (in anaerobic jar) for 48 hours. Commercial MRS agar was rehydrated in distilled water according to manufacturer's instruction. It was divided into portions of $200 \mathrm{ml}$ in a flask of $250 \mathrm{ml}$ capacity and sterilized in an autoclave at $121^{\circ} \mathrm{C}$ for 15 minutes at 15 psi. Agar was cooled down to $45^{\circ} \mathrm{C}$, poured in sterilized petri plates and allowed to solidify.

\section{Enumeration of bacteria}

The enumeration was carried out using pour plate technique on MRS medium and anaerobic jar was used for creating anaerobic conditions for 48 hours at $37^{\circ} \mathrm{C}$.
Plates with 30-300 colonies were enumerated and recorded as colony forming units $(\mathrm{CFU} / \mathrm{ml})$ of the bacterial culture.

\section{Identification of isolates}

Colonies were observed macroscopically which were obtained after 48 hours of incubation. Cultural examination included texture, size, edges and colony color. Microscopic characterization was done after Gram's staining and Lactobacillus was observed as Gram positive rods. For further identification biochemical profiling was done by citrate test, catalase test, Methyl red test and Voges Proskauer test. Lactobacillus casei and Lactobacillus lactis were used in this study. L. casei was used to optimize the composition of modified MRS medium while the L. lactis was used to validate the effectiveness of modified medium.

\section{Modification of MRS media}

Modifications in MRS media were carried out to improve its selectivity and specificity for the enumeration of $L$. casei. For this, glucose was substituted with Calcium gluconate and several concentrations were tested $(0.5,1.0,1.5$, $2.0 \mathrm{~g} / 100 \mathrm{ml})$ at $37^{\circ} \mathrm{C}$ temperature with $L$. casei and L. lactis. Media was prepared by the following method (Table 1).

Different concentrations of calcium gluconate were added $(5 \mathrm{~g}, 10 \mathrm{~g}, 15 \mathrm{~g}$, $20 \mathrm{~g} / \mathrm{L}$ ) in the above composition for the preparation of modified MRS medium. The $\mathrm{pH}$ was adjusted to $7.0 \pm 0.2$ by using $1 M \mathrm{HCl}$ and $10 \mathrm{M} \mathrm{NaOH}$. The medium was autoclaved for 15 minutes at $121^{\circ} \mathrm{C}$ before pouring. Previously isolated and identified fresh colonies of $L$. case $i$ and $L$. lactis were inoculated on different concentration of the modified MRS medium and incubated at $37^{\circ} \mathrm{C}$ anaerobically for 48 hours.

\section{Results \\ Enumeration of bacteria on modified MRS medium}

Experiments were carried out to compare the growth of L. casei and L. lactis on the modified culture media at different 
concentrations. Significant white colonies with irregular edges of $L$. casei were observed on the modified MRS medium (Fig. 1) containing $1.5 \%$ calcium gluconate as compared to $0.5,1.0$ and $2.0 \%$ concentration $/ 100 \mathrm{ml}$ of the medium while all the concentrations inhibited the growth of $L$. lactis which was unable to ferment calcium gluconate (Fig. 2). The DNA extraction and PCR based identification was carried out on all the isolates of L. casei.

Table 1. Preparation of Modified MRS media

\begin{tabular}{|c|c|}
\hline Ingredients & g/L \\
\hline Peptone & $10 \mathrm{~g}$ \\
\hline Beef extract & $10 \mathrm{~g}$ \\
\hline Yeast extract & $5 \mathrm{~g}$ \\
\hline Polysorbate 80 & $1.0 \mathrm{ml}$ \\
\hline Ammonium Citrate & $2.0 \mathrm{~g}$ \\
\hline Sodium acetate & $5.0 \mathrm{~g}$ \\
\hline Magnesium Sulfate & $0.1 \mathrm{~g}$ \\
\hline Manganese Sulphate & $0.05 \mathrm{~g}$ \\
\hline Di-potassium Phosphate & $2.0 \mathrm{~g}$ \\
\hline Agar & $15.0 \mathrm{~g}$ \\
\hline Phenol red & indicator \\
\hline
\end{tabular}

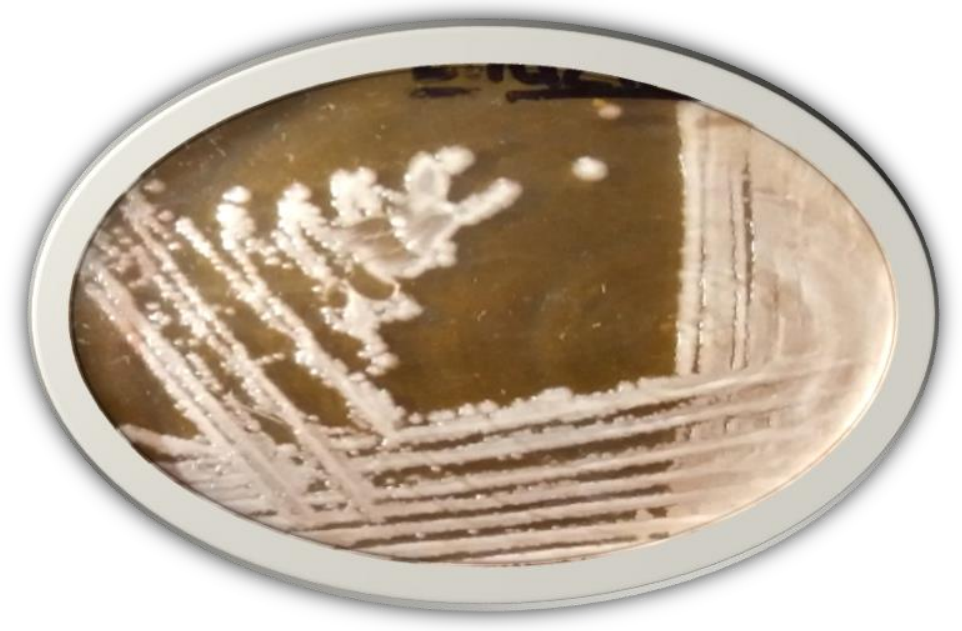

Figure 1. Growth of Lactobacillus casei on modified MRS media

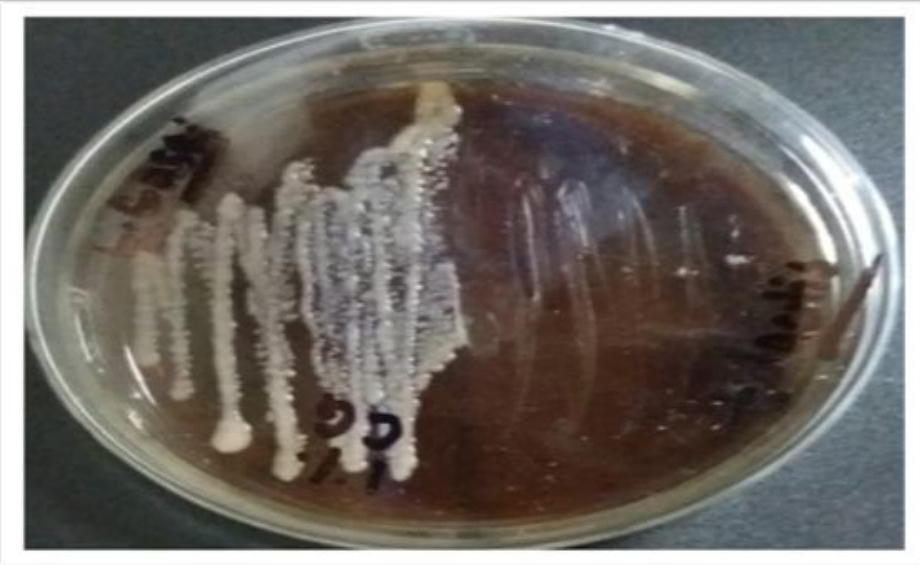

Figure 2. Growth pattern of Lactobacillus casei on one side while the inhibition of Lactobacillus lactis on other side of the modified MRS media 
Molecular identification of the isolates

Aliquots of $10 \mathrm{ng}$ of DNA extracted from the pure culture of each isolate of $L$. casei (from modified MRS medium plates) were subjected to molecular identification by PCR using 16sRNA primers. $\mathrm{F}$ : TGGATCACCTCCTTTCTA, R: GTGCGCCCTTTATTAACTT.

Thermocycling described by (Sajid et al, 2020 ) in $50 \mu 1$ reaction mixture containing $25 \mu 1$ of master mix, $2 \mu 1$ of each primer, $5 \mu 1$ of genomic DNA and $18 \mu \mathrm{l}$ of nuclease free water. Random amplification was performed using PCR conditions of initial denaturation at $94^{\circ} \mathrm{C}$ for 4 minutes, followed by 30 cycles consisted of denaturation at $94^{\circ} \mathrm{C}$ for $30 \mathrm{sec}$, annealing at $56^{\circ} \mathrm{C}$ for $30 \mathrm{sec}$, extension at $72^{\circ} \mathrm{C}$ for $45 \mathrm{sec}$ and final extension at $72^{\circ} \mathrm{C}$ for 7 minutes [10]. The resulted bands of 450bp were observed through gel documentation system after electrophoresis and Ethidium Bromide staining (Fig. 3).

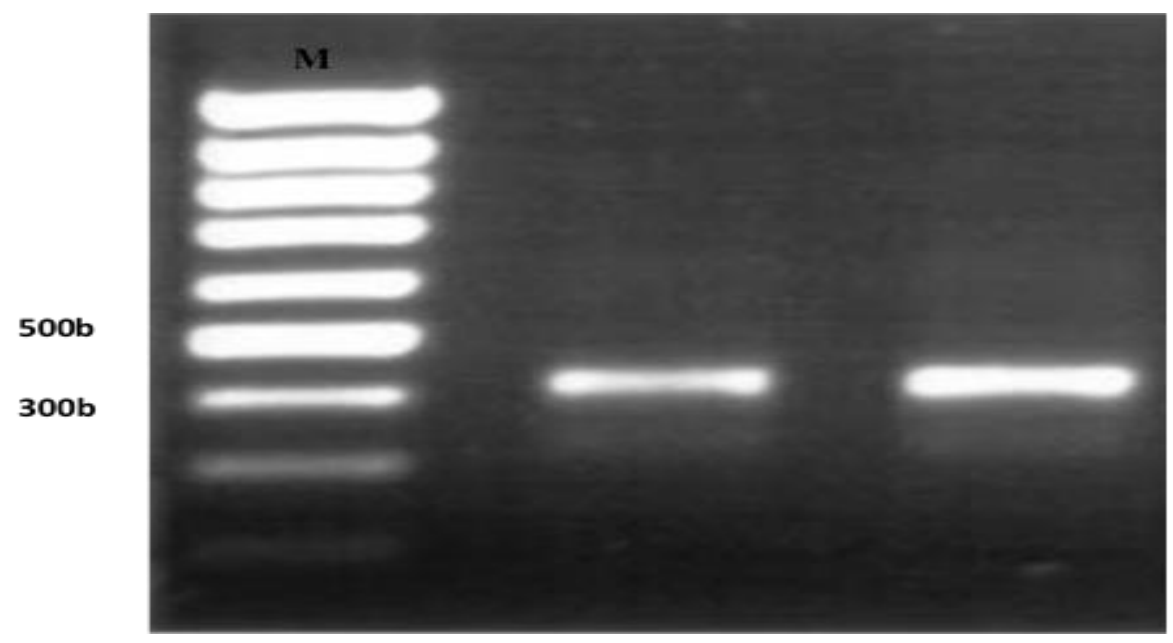

Figure 3. Molecular characterization of $L$. casei showing $450 \mathrm{bp}$ band after PCR amplification

\section{Discussion}

LAB is a fundamental group of bacteria having Gram positive property and catalase negative in nature. They produce major end product which is lactic acid and ferment carbohydrates. Lactobacilli are the most authoritative organisms in human nutrition and food microbiology. They contribute in fermentation of food products and commonly known as probiotic. According to taxonomy, Lactobacillus comes under family called Lactobacillaceae [11]. The culture of Lactobacillus is important for commercial purposes include dietary adjuncts and fermentative starters [12].

In this study, we modified the MRS medium for the reliable and selective enumeration of L. casei. Initially we assay Lactobacillus casei and Lactobacillus lactis using MRS medium. The MRS medium was chosen since it is widely recognized as an optimal medium for the growth and enumeration of Lactobacilli under both aerobic and anaerobic conditions, due to its nutritional components and acidity. On the basis of previous studies, MRS agar exhibits a low capability of discrimination among $L$. case $i$ and L. lactis. To improve the selectivity of this medium, we have modified its formulation by substituting the glucose with calcium gluconate to void the growth of $L$. lactis. The selectivity of this modified medium was tested at different concentrations of calcium gluconate with the isolated colonies of $L$. casei and L. lactis anaerobically. Thus, modified MRS with $1.5 \%$ calcium gluconate allows the optimal growth of all the isolates of $L$. casei at $37^{\circ} \mathrm{C}$ after 48 hours of incubation anaerobically but none of the L. lactis could grow in any concentration of calcium gluconate containing modified MRS medium. The concentration more than $1.5 \%$ may have deleterious effects even on the growth of $L$. casei.

Different types of bacteria which have ability to gain energy in the form of organic molecules which are not usual nutrients for 
the huge group of bacteria. For instance, $L$. casei and enterococci grow best in the presence of malate, pentitols and calcium gluconate. These organisms contain specialized and novel type of system called phosphotransferase system which metabolizes these substrates.

\section{Conclusion}

Initially Lactobacillus casei and Lactobacillus lactis was isolated using MRS medium but MRS agar exhibits a low capability of discrimination among $L$. case $i$ and $L$. lactis. To improve the selectivity of this medium, a modified MRS medium with calcium gluconate was used. Modified MRS with $1.5 \%$ calcium gluconate allows the optimal growth of all the isolates of $L$. casei at $37^{\circ} \mathrm{C}$ after 48 hours of incubation anaerobically but none of the L. lactis could grow in any concentration of calcium gluconate.

\section{Authors' contributions}

Conceived and designed the experiments: SU Rahman, Performed the experiments: I Farzand, Analyzed the data: S Sajid, Contributed materials/ analysis/ tools: S Nayab, Wrote the paper: I Farzand \& S Sajid.

\section{Acknowledgment}

The authors are thankful to the Institute of Microbiology for supporting and providing all possible facilities during research.

\section{References}

1. Azizpour K (2009). Biochemical characterization of lactic acid bacteria isolated from rainbow trout (Oncorhynchus mykiss) of West Azarbaijn. Iran. Res J Biol Sci 4(3): 324-326.

2. Puniya M, Kumar S \& Dhewa,T (2012). Isolation and biochemical characterization of Lactobacillus species isolated from Dahi. $J$ Antibiot. Res 1: 1-1p.

3. Nigatu A (2000). Evaluation of numerical analyses of RAPD and API $50 \mathrm{CH}$ patterns to differentiate Lactobacillus plantarum, Lact. fermentum, Lact. rhamnosus, Lact. sake, Lact. parabuchneri, Lact. gallinarum, Lact. casei, Weissella minor and related taxa isolated from kocho and tef. J Appl Microbiol 89(6): 969-978.

4. Sutula J, Coulthwaite LA, Thomas LV \& Verran J (2013). The effect of a commercial probiotic drink containing Lactobacillus casei strain Shirota on oral health in healthy dentate people. Microb Ecol Health Dis 24(1): 21003.

5. Tsai SY, Lin HY, Hsu YC \& Lin CP (2017). Prediction of the growth and storage conditions of Lactobacillus plantarum and Lactobacillus casei by isothermal kinetic simulation. Thermochim Acta 655: 363371.

6. Mishra V \& Prasad DN (2005). Application of in vitro methods for selection of Lactobacillus casei strains as potential probiotics. Int $J$ Food Microbiol 103(1): 109-115.

7. Klerk HC \& Coetzee JN (1962). Secondary Colony Formation by Lactobacillus casei. Microbiol 29(2): 291-299.

8. Reuter, G (1985). Elective and selective media for lactic acid bacteria. Int $J$ Food Microbiol 2(1-2): 55-68.

9. Noori F, Tajabafi EM \& Jafari P (2016). Growth Optimization of Lactobacillus plantarum T5jq301796. 1, an Iranian Indigenous Probiotic in Lab Scale Fermenter. Appl Food Biotechnol 3(3):188-193.

10. Sajid S, Rahman SU, Nayab S, Khan IU \& Javeed A (2020). Infectious bursal disease virus cloning and structural protein (VP2) expression in Escherichia coli. PAB 9(1): 743-749.

11. Salvetti E, Torriani $S$ \& Felis GE (2012). The genus Lactobacillus: a taxonomic update. Probiotic Antimicro 4(4): 217-226.

12. Wright CT \& Klaenhammer T (1981). Calcium-induced alteration of cellular morphology affecting the resistance of Lactobacillus acidophilus to freezing. Appl Environ Microbiol 41(3): 807-815. 